\title{
Lack of Primary Care in Rural Areas for People with Disabilities
}

\author{
Knicole Lee* \\ College of Nursing and Health Sciences, Valdosta State University, USA
}

*Corresponding author: Knicole Lee, College of Nursing and Health Sciences, Valdosta State University, Georgia, USA.

Received Date: November 08, 2019

Published Date: March 18, 2020

\section{Mini Review}

People residing in rural areas face difficulties in accessing primary medical care, more so when they have disabilities. This paper seeks to identify the reasons why people with disabilities have difficulty acquiring medical and social services. In addition, outlined will be the difficulties and effects brought about by the neglect, and finally, establish solutions that will aid in improving access to medical care by people with disabilities in the rural setups. According to Lishner, Richardson and Levine (47) [1], the major factors that hinder access to medical care in the rural areas include difficulty in attracting and retaining qualified health care providers, lack of training of primary care providers on how to treat complex needs associated with disability, and lack of resources required to offer specialized services. In addition, rural areas have a low population density and are separated by time and distance from urban areas. This places rural areas at a disadvantaged when it comes to accessing medical care and resources. Jones argues that even if there was an equal distribution of medical care, people rural areas would still face difficulty in accessing the resources due to geographic barriers of time and distance (32).

Poor infrastructure and bad environmental conditions also affect the ability of people in the rural areas to access timely medical care. The effects of the weather and poor infrastructure get worse if the population is poor, as the poor cannot afford private cars or expensive air travel expenses. This means that they will have to rely on public transport, which is always unreliable and limited. This factor has a direct link to the economic status that widely acts as a health determinant. Rural areas tend to have lower and poor economy as opposed to urban settings.
Starfield, Shi, and Macinko (485) [2] argues that countries have a "strong" basic primary care system tend to achieve better healthcare outcomes. Improved health outcome is also achieved at a lower cost than countries characterized by weaker primary care base. The absence of a policy to take health personnel and facilities to areas of greatest needs leads to a concentration of these resources in areas that need them least because such areas that are mostly in a position to pay for those facilities.

Health facilities in the rural area have fewer personnel. This is a major cause of lack of access to health care. Lack of health providers with appropriate comprehension in areas like rare disabilities and low incidence disabilities reduces the level of care that they can offer to rural populations. In addition, patients in rural areas lack proper facilities, equipment, and physicians who have the technological knowhow to cure conditions that require computerized systems [3]. Rural areas have no rehabilitation centers or physical, speech, and occupational therapists. Moreover, the poor coordination of care facilities between the urban and rural areas makes it difficult for disabled patients from the rural areas to get treatment from urban physicians.

Many hospitals have developed the habit of dumping patients with special needs. Dumping refers to premature discharge of patients to other health delivery system for care [4]. Rural health care systems dump the highest number of patients to large hospitals. The large number of people dumbed in health facilities in the urban areas burdens the small health care systems. However, the health facilities in urban areas do not have the capability to 
take care of these patients because they are understaffed, have less medical equipment, and capital to take care of that many patients, which leads to a total failure of the system.

Inadequate information and communication channels to deliver information to rural areas are one leading factor in primary health accessibility. Moore Robert maintains that most health care facilities lack professional interpreters for deaf individuals (184). Robert further argues that there are no medium to provide information to people with disability about the existing programs, the requirements for eligibility, and how to access the available services. It is quite apparent that public awareness and education vis-à-vis the needs of individuals with disabilities is little or not present at all. Robert's study makes it clear that the people with disabilities know very little about health care services for the disabled or how to access them.

The above-mentioned factors show that people with disabilities in rural areas are facing real hurdles with health care services. To counter these problems sanctions should be put in place to discourage dumping. Hospitals should have proper and working facilities to take care of patients until they fully recover. In case of a referral to rural health systems, the systems should be well equipped with equipment, facilities, and health providers to take care of the disabled.

To increase health care, the health system needs to provide information about their services. Substitute locations need to be put up for care and communication. Salons, coffee shops, beauty salons, and offices could act as alternative sites where people with disabilities can access information and health care services. This will help reduce the number of people who do not have access to primary medical care. Financial constraints are major reasons for people with disabilities no to access health care services. Medical equipment and facilities are expensive. In addition, transport costs and insurance covers are usually too high for most disabled people. Thus, the government should determine how to provide the equipment and services that the disabled require at a reasonable price. People with disabilities also need centers that will provide a platform to socialize, so that they can get information from different individuals. Such platforms act not only as entertainment forums, but also, as educative, informative, and networking mediums.

The advancement of technology is proving to be a positive factor to rural settings. Telemedicine involves the use of electronic information and telecommunication technologies in health care [58]. This technology allows health care providers in rural settings to communicate with their urban counterparts in cases of complex medical conditions. Telemedicine will make it easy to reach most of the individuals in rural areas. Finally, the message dispersed will be tailored depending on the user's disability.

In conclusion, rural areas lack primary health care for people with disabilities. As much as the rural economy, geographic distribution, and infrastructure contribute to this, the main problem lies in the health care system itself. Their policies need a quick review to accommodate and cater for people in the population. They should not be ignored just because the population is small. People with disabilities are entitled to good health care services regardless of the setting they decide to settle in. The health care system should strive to improve medical care and bring it as close to the disabled as possible.

\section{Acknowledgement}

None.

\section{Conflict of Interest.}

No conflict of interest.

\section{References}

1. Lishner DM, Richardson M, Levine P, Patrick D (1996) Access to Primary Health Care Among Persons with Disabilities in Rural Areas: A Summary of the Literature. The Journal of Rural Health 12(1): 45-53.

2. Starfield Barbara, Leiyu Shi, James Macinko (2005) Contribution of Primary Care to Health Systems and Health. Milbank Q 83(3): 47-52.

3. Zejda Jan E, Helen H McDuffie, James A Dosman (1993) Epidemiology of Health and Safety Risk in Agriculture and Related Industries: Practical Applications for Rural Physicians. The western Journal of Medicine 158(1): 56-63.

4. Fabrikant, Robert (2016) Health Care Fraud: Enforcement and Compliance. NY: Law Journal Press, New York.

5. Gulla, Vincenzo (2013) Telehealth Networks for Hospital Services: New Methodologies. Hershey PA: Medical Information Science Reference, Pennsylvania.

6. Donham, Kelley J, Kim M Thu (1993) Relationships of Agricultural and Economic Policy to The Health of Farm Families, Livestock and the Environment. J Am Vet Med Assoc 202(7): 1084-1091.

7. Jones Roger (2004) Oxford Textbook of Primary Medical Care. New York: Oxford University Press, USA.

8. Robert Moore (2001) The Hidden America: Social Problems in Rural America for the Twenty-first Century. London: Susquehanna University Press, UK. 